\title{
Risk Factors for Deep-Vein Thrombosis and Pulmonary Thromboembolism in Benign Ovarian Tumor
}

\author{
Mitsuru Shiota, ${ }^{1}$ Yasushi Kotani, ${ }^{1}$ Masahiko Umemoto, ${ }^{1}$ Takako Tobiume, ${ }^{1}$ \\ Mitsuhiro Tsuritani, ${ }^{1}$ Masao Shimaoka ${ }^{1}$ and Hiroshi Hoshiai ${ }^{1}$
}

${ }^{1}$ Department of Obstetrics and Gynecology, Kinki University Faculty of Medicine, Osaka, Japan

\begin{abstract}
Pulmonary thromboembolism (PE) is a serious postoperative complication. Reported rates of PE following gynecologic surgery are between $0.3 \%$ and $0.8 \%$, with deep-vein thrombosis (DVT) as the major cause (via seeding of the lungs). Benign ovarian tumors are treated principally by surgery. Possible risk factors for DVT and PE in patients with benign ovarian tumors include tumor size, patient age, and obesity. To date, however, there has been no report addressing the association of these risk factors in patients with benign ovarian tumors. This study offers a retrospective analysis of the incident of preoperative DVT by age, tumor size, and BMI in patients undergoing surgery for benign ovarian tumors. A total of 843 Japanese patients with a preoperative diagnosis of benign ovarian tumor who underwent tumorectomy or adnexectomy at our institution between July 2003 and December 2010 were enrolled. The incidence of preoperative DVT was monitored and statistically stratified by age ( $<50$ years and $\geq 50$ years), largest tumor diameter $(<10 \mathrm{~cm}$ and $\geq 10 \mathrm{~cm})$, and $\mathrm{BMI}(<25$ and $\geq 25)$. The result indicates that tumor diameter of $\geq 10 \mathrm{~cm}$ is not a risk factor for preoperative DVT in patients with benign ovarian tumor. On the other hand, age $\geq 50$ years and BMI $>25$ are independent risk factors for preoperative DVT in Japan. The patients with each risk factor should be treated with preoperative, intraoperative, and postoperative precautions against development of PE.
\end{abstract}

Keywords: d-dimer; deep-vein thrombosis; ovarian tumor; postoperative complication; pulmonary thromboembolism Tohoku J. Exp. Med., 2011, 225 (1), 1-3. ㄷ 2011 Tohoku University Medical Press

Benign ovarian tumor, one of the most common gynecologic conditions, is treated principally by surgery. These tumors are most often treated laparoscopically, and the definitive diagnosis is provided by post-operative histopathology. With recent advances in laparoscopic techniques, laparoscopic surgery has become the preferred mode of treatment for benign ovarian tumors, with benefits including decreased postoperative pain and a shorter period of convalescence. However, laparoscopic surgery can still be accompanied by serious complications, including pulmonary thromboembolism (PE).

$\mathrm{PE}$ is a dire complication, often leading to shock or sudden death. In the presence of undiagnosed deep vein thrombosis (DVT), PE secondary to seeding of the lungs may occur upon a patient's first postoperative attempt at ambulation. DVT is diagnosed postoperatively in $17 \%$ to $20 \%$ of gynecological surgery patients (Nicolaides 1997; Geerts et al. 2001; Sakon et al. 2006), and the reported rate of PE following gynecologic surgery is between $0.3 \%$ and $0.8 \%$. Moreover, PE and deep vein thrombosis (DVT) are estimated to occur in $0.02 \%$ of all laparoscopic gynecologic surgeries (Jorgensen et al. 1994).
Malignancy, aging, and obesity are among the factors that have been reported to increase the risk of DVT in patients undergoing gynecologic surgery (Anderson et al. 2003). Suzuki et al. (2010) have suggested that ovarian cancers $\geq 10 \mathrm{~cm}$ in diameter and the history of previous venous thromboembolic episodes might be pertinent risk factors. Shiota et al. (2011) have reported that in patients with benign uterine fibroids, larger tumor diameter is associated with increased risk of DVT, most likely because of venous compression by the mass. Age and obesity were not related to the incidence of DVT in these patients.

The risk of postoperative PE can be proactively reduced through the proper preoperative diagnosis and treatment of asymptomatic DVT. Knecht et al. (1997) have found that acute venous thrombosis can be effectively excluded by D-dimer levels below a reference value of 0.5 $\mu \mathrm{g} / \mathrm{ml}$. D-dimer is generated when a fibrin clot is dissolved by plasmin. Its elevated levels are thus suggestive of disseminated intravascular coagulation (DIC) or thromboembolism. D-dimer levels are essential for detection of asymptomatic DVT (Greenfield et al. 1993; Tajima et al. 2004), and can be used in screening for preoperative DVT

Received May 19, 2011; revision accepted for publication July 26, 2011. doi: 10.1620/tjem.225.1

Correspondence: Mitsuru Shiota, Department of Obstetrics and Gynecology, Kinki University Faculty of Medicine, 377-2 Ohno-higashi,

Osaka-sayama, Osaka, 589-8511, Japan.

e-mail: shiota@med.kindai.ac.jp 
as a measure to prevent PE.

The current study focuses on the incidence of preoperative DVT in patients with benign ovarian tumors. The authors have not found any previous reports that include statistical analyses of the risk of DVT and PE in relation to tumor size, patient age, and BMI for patients with benign ovarian tumors. We have thus investigated the relationship between these factors and the occurrence of preoperative DVT in these patients.

\section{Materials and Methods}

A total of 843 patients (laparoscopic surgery 740 cases and open surgery 103 cases) with preoperative diagnoses of benign ovarian tumor who underwent tumorectomy or adnexectomy in a single hospital between July 2003 and December 2010 were enrolled. Preoperative ovarian tumor diagnoses were based on methods including MR and ultrasound imaging and assay for tumor makers. All relevant ethical safeguards including the Declaration of Helsinki have been met in relation to patient or subject protection.

Patients were screened for DVT preoperatively by D-dimer assay, which has been routine for all surgical candidates at our hospital since July 2003. A two-step sandwich-type enzyme immunoassay (EIA) (D-dimer assay "Kokusai", Sysmex Corp., Japan) was performed on sodium citrate-added blood samples for the D-dimer measurements. A value of $0.5 \mu \mathrm{g} / \mathrm{ml}$ is used as the reference value because the true negative rate for DVT and PE below that value is over $99 \%$.

For patients with D-dimer levels exceeding $0.5 \mu \mathrm{g} / \mathrm{ml}$, lower extremity Doppler ultrasound using a 5- to $10-\mathrm{MHz}$ linear electronic probe was performed with the patient either seated or supine with slight leg abduction. If Doppler scan for DVT was negative, intraand postoperative compression stockings and intraoperative intermittent pneumatic compression (IPC) were implemented. If the Doppler scan was positive for DVT, intra- and postoperative compression stockings were used, but IPC was excluded. Heparin sodium was intravenusly administered preoperatively and for 5-7 days postoperatively for the patients with confirmed DVT. The starting dose of 12,000 units/day was titrated until the activated partial thromboplastin time (aPTT) reached 1.5 to 2.5 times the control.

Accrued patient data in this study included age, largest tumor diameter as measured by MRI or ultrasound, BMI, D-dimer level, D-dimer positive $(\geq 0.5 \mu \mathrm{g} / \mathrm{ml})$ rate, and incidence of DVT. DVT rates were compared for patient ages $<50$ years and $\geq 50$ years, for largest tumor diameter $<10 \mathrm{~cm}$ and $\geq 10 \mathrm{~cm}$, and for $\mathrm{BMI}<25$ and $\geq 25$.

Results were subject to Chi-Square analysis, with a $P$-value $<0.05$ indicating statistical significance.

\section{Results}

Mean patient characteristics were as follows: age 38.4 years; largest tumor diameter $7.0 \mathrm{~cm}$; BMI 21.4; and D-dimer level $0.36 \mu \mathrm{g} / \mathrm{ml}$ (Table 1). Overall, $16.6 \%$ $(140 / 843)$ of the patients had preoperative D-dimer levels $\geq 0.5 \mu \mathrm{g} / \mathrm{ml}$, and $3.7 \%(31 / 843)$ had DVT confirmed by ultrasound.

The incidence of preoperative DVT was $1.6 \%(11 / 699)$ in patients $<50$ years of age and $13.9 \%(20 / 144)$ in patients $\geq 50$ years of age, a statistically significant variation. The rate of preoperative DVT was significantly higher in patients with $\mathrm{BMI} \geq 25(10.6 \%(11 / 104)$ versus $0.3 \%$ $(20 / 739)$ in patients with BMI $<25)$. The incidence of preoperative DVT was 3.3\% (23/690) for largest tumor diameter $<10 \mathrm{~cm}$ and $5.2 \%(8 / 153)$ for largest tumor diameter $\geq 10 \mathrm{~cm}$. This difference was not statistically significant. There was no significant difference observed in DVT incidence by tissue type in this study.

\section{Discussion}

There was no correlation found between tumor size or tissue type and incidence of preoperative DVT in this study of patients undergoing surgery for benign ovarian tumor. The pathogenesis of DVT in gynecologic surgery patients conforms to the classic triad of Virchow (1854): stasis, hypercoagulability, and vessel wall abnormality. Some malignancies are known to increase coaguability, and DVT/ $\mathrm{PE}$ has been reported in patients with ovarian cancer (Suzuki et al. 2010). An increased risk of DVT has also

Table 1. Patient Characteristics.

\begin{tabular}{lc}
\hline \multicolumn{1}{c}{ Characteristic } & $\begin{array}{c}\text { Mean } \pm \text { s.d. } \\
\text { (range) }\end{array}$ \\
\hline Age (years) & $38.4 \pm 14.1$ \\
& $(12-86)$ \\
Largest tumor diameter $(\mathrm{cm})$ & $7.0 \pm 3.5$ \\
BMI & $(1-30)$ \\
& $21.4 \pm 3.4$ \\
D-dimer $(\mu \mathrm{g} / \mathrm{ml})$ & $(13.5-53.3)$ \\
& $0.36 \pm 0.51$ \\
D-dimer positive rate & $(0.04-8.57)$ \\
DVT incidence & $16.6 \%(140 / 843)$ \\
\hline
\end{tabular}

Table 2. DVT Rate by Category.

\begin{tabular}{lcc}
\hline \multicolumn{1}{c}{ Category } & DVT Rate & $P$ value \\
\hline Age $<50$ & $1.6 \%(11 / 699)$ & $P<0.01$ \\
Age $\geq 50$ & $13.9 \%(20 / 144)$ & \\
Largest tumor diameter $<10 \mathrm{~cm}$ & $3.3 \%(23 / 690)$ & $P=0.26$ \\
Largest tumor diameter $\geq 10 \mathrm{~cm}$ & $5.2 \%(8 / 153)$ & \\
BMI $<25$ & $0.3 \%(20 / 739)$ & $P<0.01$ \\
BMI $\geq 25$ & $10.6 \%(11 / 104)$ & \\
\hline
\end{tabular}


been demonstrated in patients with large uterine fibroids, possibly due to compression of adjacent blood vessels including the inferior vena cava (Shiota et al. 2011).

Suzuki et al. (2010) have reported an incidence rate of $3.17 \%$ for perioperative DVT in women with surgical gynecological disease, including patients with malignant tumors. In patients with benign gynecologic diseases, such as uterine fibroids, the size of the mass has been found to be a factor for increased risk of DVT (Shiota et al. 2011). If these large masses compress the inferior vena cava and other pelvic veins, the resulting venous stasis may lead to DVT (Shiota et al. 2011).

This report has examined the relationship of tumor size, patient age, and BMI with DVT risk in patients undergoing surgery for benign ovarian tumors. Positive correlation with increased DVT risk were found for age $>50$ years and BMI $>25$ in this study population. The absence of correlation between tumor size or tissue type and the incidence of preoperative DVT in these patients may be because benign ovarian tumors are not as likely as heavier uterine fibroids to compress the IVC or other pelvic vessels.

Age and obesity have previously been determined to be risk factors in the development of DVT/PE (Anderson et al. 2003) in gynecologic surgery. In this study, DVT was diagnosed preoperatively in $13.9 \%$ of patients $>50$ years old and in $10.6 \%$ of patients with BMI $>25$. The BMIrelated risk of DVT would skew much lower with obesity defined by BMI $\geq 30$, but most reports define obesity in Asian populations as BMI $>25$ (Ko et al. 1999). Therefore, in this study, BMI $>25$ was determined to be a significant risk factor for preoperative DVT. It is recommended that preoperative DVT status be determined and prophylactic measures against PE be implemented for all patients, particularly when age is $>50$ years and/or BMI is $>25$.

Preventive measures for PE during the perioperative period can include intra- and postoperative compression stockings and intraoperative intermittent pneumatic compression (IPC) for patients without DVT. For patients with DVT, IPC is withheld, but intra- and postoperative compression stockings can be used and I.V. heparin sodium administered preoperatively and for 5-7 days postoperatively in a dose adjusted for an aPTT of 1.5 to 2.5 times control (Holford 1976; Karmy-Jones et al. 2007; Kim et al. 2008).

\section{Conclusion}

In patients with benign ovarian tumor, tumor size of $>10 \mathrm{~cm}$ is not a risk factor for DVT, but age $>50$ years and BMI $>25$ are independent risk factors. Preventive measures for PE are recommended for all patients, and particularly for patients with these risk factors.

\section{Conflict of Interest}

All authors in this study are free of conflict of interest.

\section{References}

Anderson, F.A. Jr. \& Spencer, F.A. (2003) Risk Factors for Venous Thromboembolism. Circulation, 107, I-9-I-16.

Geerts, W.H., Heit, J.A., Clagett, G.P., Pineo, G.F., Colwell, C.W., Anderson, F.A. Jr. \& Wheeler, H.B. (2001) Prevention of venous thromboembolism. Chest, 119, 132S-175S.

Greenfield, L.J., Proctor, M.C., Williams, D.M. \& Wakefield, T.W. (1993) Long-term experience with transvenous catheter pulmonary embolectomy. J. Vasc. Surg., 18, 450-458.

Holford, C.P. (1976) Graded compression for preventing deep venous thrombosis. Br. Med. J., 23, 969-970.

Jorgensen, J.O., Lalak, N.J., North, L., Hanel, K., Hunt, D.R. \& Morris, D.L. (1994) Venous stasis during laparoscopic cholecystectomy. Surg. Laparosc. Endosc., 4, 128-133.

Karmy-Jones, R., Jurkovich, G.J., Velmahos, G.C., Burdick, T., Spaniolas, K., Todd, S.R., McNally, M., Jacoby, R.C., Link, D., Janczyk, R.J., Ivascu, F.A., McCann, M., Obeid, F., Hoff, WS., McQuay, N. Jr., Tieu, B.H., Schreiber, M.A., Nirula, R., Brasel, K., Dunn, J.A., Gambrell, D., Huckfeldt, R., Harper, J., Schaffer, K.B., Tominaga, G.T., Vinces, F.Y., Sperling, D., Hoyt, D., Coimbra, R., Rosengart, M.R., Forsythe, R., Cothren, C., Moore, E.E., Haut, E.R., Hayanga, A.J., Hird, L., White, C., Grossman, J., Nagy, K., Livaudais, W., Wood, R., Zengerink, I. \& Kortbeek, J.B. (2007) Practice patterns and outcomes of retrievable vena cava filters in trauma patients: an AAST Multicenter Study. J. Trauma, 62, 17-25.

Kim, H.S., Young, M.J., Narayan, A.K., Hong, K., Liddell, R.P. \& Streiff, M.B. (2008) A comparison of clinical outcomes with retrievable and permanent inferior vena cava filters. J. Vasc. Interv. Radiol., 19, 393-399.

Knecht, M.F. \& Heinrich, F. (1997) Clinical evaluation of an immunoturbidimetric D-dimer assay in the diagnostic procedure of deep vein thrombosis and pulmonary embolism. Thromb. Res., 88, 413-417.

Ko, G.T., Chan, J.C., Cockram, C.S. \& Woo, J. (1999) Prediction of hypertension, diabetes, dyslipidaemia or albuminuria using simple anthropometric indexes in Hong Kong Chinese. Int. J. Obes. Relat. Metab. Disord., 23, 1136-1142.

Nicolaides, A.N. (1997) International Consensus Statement (Guidelines compiled in accordance with the scientific evidence)., vol. 16, no. 1.

Sakon, M., Maehara, Y., Yoshikawa, H. \& Akaza, H. (2006) Incidence of venous thromboembolism following major abdominal surgery: a multi-center, prospective epidemiological study in Japan. J. Thromb. Haemost., 4, 581-586.

Shiota, M., Kotani, Y., Umemoto, M., Tobiume, T., Tsuritani, M., Shimaoka, M. \& Hoshiai, H. (2011) Deep-vein thrombosis is associated with large uterine fibroid. Tohoku J. Ex. Med., 224, 87-89.

Suzuki, N., Yoshioka, N., Ohara, T., Yokomichi, N., Nako, T., Yahagi, N., Igarashi, S., Kobayashi, Y., Yoshimatsu, M., Takizawa, K., Nakajima, Y., Kiguchi, K. \& Ishizuka, B. (2010) Risk factors for perioperative venous thromboembolism: a retrospective study in Japanese women with gynecologic diseases. Thromb. J., 8, 17.

Tajima, H., Murata, S., Kumazaki, T., Nakazawa, K., Kawamata, H., Fukunaga, T., Yamamoto, T., Tanaka, K. \& Takano, T. (2004) Manual aspiration thrombectomy with a standard PTCA guiding catheter for treatment of acute massive pulmonary thromboembolism. Radiat. Med., 22, 168-172.

Virchow, R. (1854) Handbuch der Speciellen Pathologie und Therapie. Vol. 11. Erlangen, F Enke. 